\title{
Influence of different beverages on the force degradation of intermaxillary elastics: an in vitro study
}

\author{
Jorge César Borges LEÃO FILHO'1, Daphine Beatriz GALLO², Regis Meller SANTANA', Odilon GUARIZA-FILHO², \\ Elisa Souza CAMARGO ${ }^{4}$, Orlando Motohiro TANAKA ${ }^{5}$
}

\author{
1- DDS, Dentistry Graduate Program - Orthodontics, Pontifical Catholic University of Paraná, Curitiba, PR, Brazil. \\ 2- Undergraduate student in Dentistry, School of Health and Biosciences, Pontifical Catholic University of Paraná, Curitiba, PR, Brazil. \\ 3- DDS, MSc, PhD, Senior Professor, Graduate Dentistry Program - Orthodontics, Pontifical Catholic University of Paraná, Curitiba, PR, Brazil. \\ 4- DDS, MSc, PhD, Associate Professor, Graduate Dentistry Program - Orthodontics, Pontifical Catholic University of Paraná, Curitiba, PR, Brazil. \\ 5- DDS, MSc, PhD, Full Professor, Graduate Dentistry Program, Orthodontics, Pontifical Catholic University of Paraná, Curitiba, PR, Brazil.
}

Corresponding address: Prof. Dr. Orlando Motohiro Tanaka - PUCPR, Programa de Pós-Graduação em Odontologia - Ortodontia - R. Imaculada Conceição, 1155 - 80215-901 - Curitiba - PR - Brasil - Phone: 5541 3271-1637 - Fax: 5541 3271-1405 - e-mail: tanakaom@gmail.com

Received: March 23, 2012 - Modification: January 4, 2013 - Accepted: January 30, 2013

\section{ABSTRACT}

O bjective: The aim of this study was to evaluate in vitro the effects of frequently ingested beverages on force degradation of intermaxillary elastics. Material and Methods: One hundred and eighty 1/4-inch intermaxillary elastics (TP Orthodontics) were immersed into six different beverages: (1) Coca-Cola ${ }^{\oplus}$; (2) Beer; (3) Orange juice; (4) Red wine; (5) Coffee and (6) artificial saliva (control). The period of immersion was 15 min for the first and second cycles and $30 \mathrm{~min}$ for the third to fifth cycles. Tensile forces were read in a tensile testing machine before and after the five immersion cycles. One-way repeated measures ANOVA was used to identify significant differences. Results: Force degradation was seen in all evaluated groups and at all observation periods $(p<0.05)$. A greater degree of degradation was present at the initial periods, decreasing gradually over time. However, no statistically significant differences were seen among groups at the same periods, showing that different groups behaved similarly. Conclusion: The chemical nature of the evaluated beverages was not able to influence the degree of force degradation at all observation periods.

Key words: Tensile strength. Beverages. Latex. Rubber.

\section{INTRODUCTION}

The use of intermaxillary elastics is a common practice in current orthodontics. These materials are made of either latex (natural rubber) or synthetic polymer materials (polyurethane). Latex elastics are more commonly used because they present improved properties such as: greater flexibility, lower cost and greater capacity of returning to the original dimensions after undergoing deformation ${ }^{14,15}$.

In orthodontic practice, the use of elastics has some advantages, such as biocompatibility, low cost, easy installation and removal by the patients ${ }^{9}$. However, the oral environment may interfere negatively in the properties of these materials.
Exposure of these elastics to oral environment results is degradation of their elastic forces. Literature has reported that rubber elastics exposed to oral environment lose between 10 to $40 \%$ of their initial force $e^{1,3,4,12,15}$. After 1211 to $24 \mathrm{~h}^{1,3,4,12,15}$ of installation, this degradation rate is greatly reduced, resulting in forces with reasonably constant intensity. Moreover, in spite of being considered a biocompatible material, Santos, et al. ${ }^{10}$ (2010) found that this material presents cytotoxicity.

The elongation of the molecular chains of materials should be considered one of the main mechanisms by which the elastic force decay occurs. When the elastic stretches, the polymer chains are uncoiled becoming elongated. If the force is excessive, such chains will slide one over another resulting in permanent deformation of the material 
and consequently in the degradation of the force generated by the elastic ${ }^{13}$.

Additionally, the oral environment acts as an agent of the elastic force degradation because of physical damages, e.g.: mechanical traumas and temperature alterations ${ }^{15}$. Moreover, the chemical interactions among the material, saliva and dietary substances promote both the absorption of water and other salivary constituents and the solubilization/release of the material's components in the saliva, consequently modifying the elastics' molecular structure and favoring their degradation ${ }^{4}$.

Most studies on the influence of the oral environment are conducted in vitro, evaluating humidity, saliva composition, $\mathrm{pH}$, temperature and stress induced by elastic cyclic stretch. However, few studies on the effects of dietary substances are found in the literature.

Beattie, et al.2 (2004) evaluated the effect of diet on the mechanical properties of intermaxillary elastics. These authors observed that dietary substances did not significantly increase the degradation of the mechanical properties of elastics. Nattrass, et al. ${ }^{8}$ (1998) verified that the immersion of elastomeric chains in Coca-Cola ${ }^{\circledR}$ resulted in a greater degradation of forces compared with the immersion in water. However, Teixeira, et al. ${ }^{14}$ (2008) demonstrated that immersion in CocaCola $^{\circledR}$ was not able to alter the pattern of force degradation in two different types of elastomeric chains.

Intermaxillary elastics have been routinely employed in orthodontics it is thus important that orthodontists be aware of the mechanical properties of these materials as well as of the influence of oral environment factors on their properties. Due to the limited number of studies evaluating the effect of diet on force degradation, the aim of this study was to evaluate in vitro the effects of frequently ingested beverages on the force degradation of intermaxillary elastics.

\section{MATERIAL AND METHODS}

One hundred and eighty medium force 1/4-inch intermaxillary elastics (Tru-Force Latex Elastic System; TP Orthodontics Inc., La Porte, IN, USA) were used and divided into six groups $(n=30)$ : group 1 (Control - artificial saliva), group 2 (CocaCola ${ }^{\circledR}$ - Coca-Cola Company, São Paulo, SP, Brazil), group 3 (Beer - Heineken, São Paulo, SP, Brazil), group 4 (orange juice - Del Valle Pure Squeezed Orange Juice, Coca-Cola Company, São Paulo, SP, Brazil), group 5 (Red wine - Santa Lucia Cabernet Sauvignon, Henriquez Hermanos Ltd., Talca, Chile), group 6 (Coffee - Nescafé Classic, Nestlé S.A., Araraquara, SP, Brazil). The composition of artificial saliva was $0.0625 \% \mathrm{KCl}, 0.0865 \% \mathrm{NaCl}$,
$0.0056 \% \mathrm{MgCl}_{2}, 0.0166 \% \mathrm{CaCl}_{2}, 0.0804 \% \mathrm{Na}_{2} \mathrm{HPO}_{4}$, $0.0326 \% \mathrm{KH}_{2} \mathrm{PO}_{4}, 4.274 \%$ sorbitol, $0.0004 \% \mathrm{NaF}$, $0.1 \% \mathrm{C}_{6} \mathrm{H}_{5}$ COONa, $2 \%$ carboxymethylcellulose and distilled water.

Elastics with tensile strength ranging from 1.55 to $2 \mathrm{~N}$ were selected to standardize the samples and eliminate possible mechanical behavior bias. Therefore, 282 elastics were tested to reach a test sample size of 180 elastics.

Initially all elastics were mounted on an epoxy resin board on two steel pins at a distance of 26 $\mathrm{mm}$ (Figures $1 \mathrm{~A}$ and $1 \mathrm{~B}$ ) and their forces were measured in a tensile testing machine (DL1000; EMIC Ltda., São José dos Pinhais, PR, Brazil) at a crosshead speed of $150 \mathrm{~mm} / \mathrm{min}$. These values $(\mathrm{N})$ were recorded as baseline reading (TO).

Next, the samples were randomly divided into six groups. The test of variance homogeneity was applied to verify the baseline forces, showing statistically similar mean values of initial forces ( $p>0.05)$ among the groups.

After the baseline readings, the elastics were immersed in a glass flask $(15 \times 9 \times 14 \mathrm{~cm})$ containing $300 \mathrm{~mL}$ of the tested beverages, according to the experimental groups. Immersion period was $15 \mathrm{~min}$ for the first (T1) and second (T2) cycles and 30 min for third to fifth (T3, T4, T5) cycles. At every immersion cycle, the beverages were changed (Figure 2) and the elastics were washed in distilled water and immersed into $300 \mathrm{~mL}$ of artificial saliva for $3 \mathrm{~min}$. Next, the elastics were again washed in distilled water and removed from the resin board to perform a new tensile strength test.

Data were analyzed by one-way repeated measures ANOVA to compare the effects of intervals, beverages and the interaction between them. The level of significance was set at $5 \%$.

\section{RESULTS}

Figure 3 shows the homogenous distribution of the sample at T0, where the groups presented
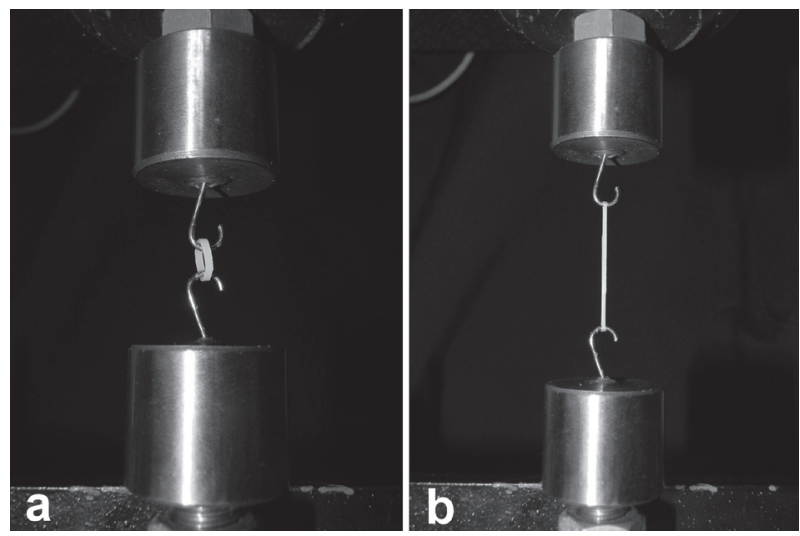

Figure 1- Tensile strength test of intermaxillary elastics. a) Elastic at rest position. b) Elastic stretched at $26 \mathrm{~mm}$ 

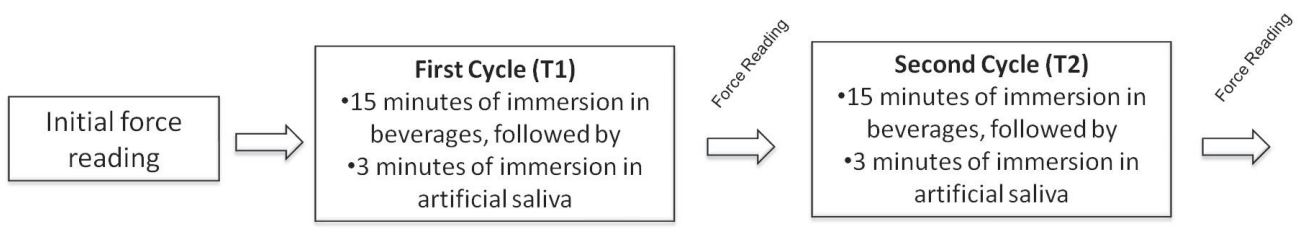
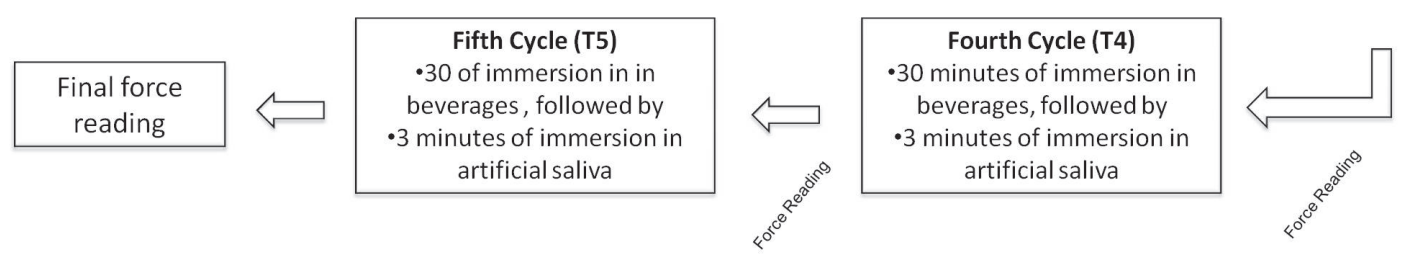

Figure 2- Sequence of the experimental groups

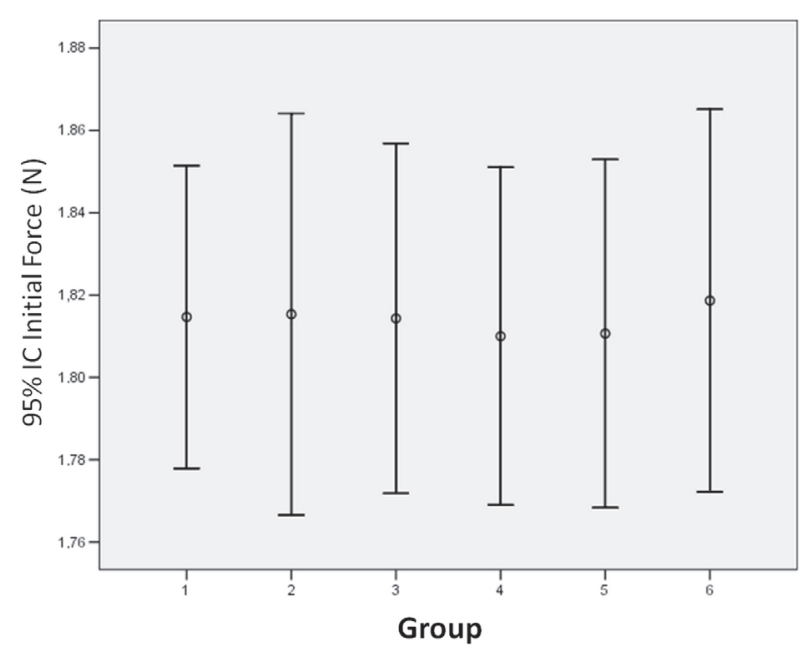

Figure 3- Homogeneous distribution of samples at T0

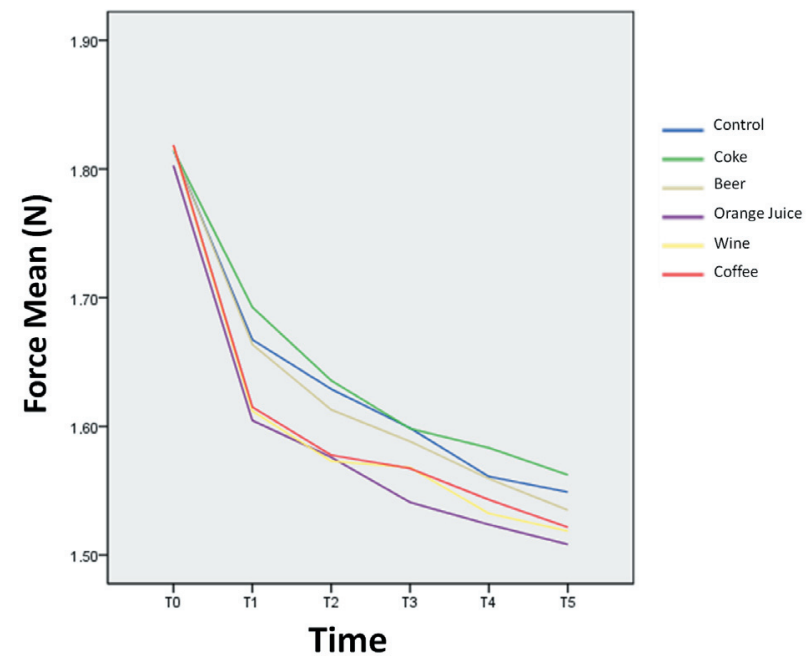

Figure 5- Mean force values of the intermaxillary elastics of each group over time

statistically similar mean values of initial force $(p>0.05)$. Since the mean force values of each group were statistically similar, it may be affirmed that the alterations were due to the tested treatments and not to the differences in each group behavior.

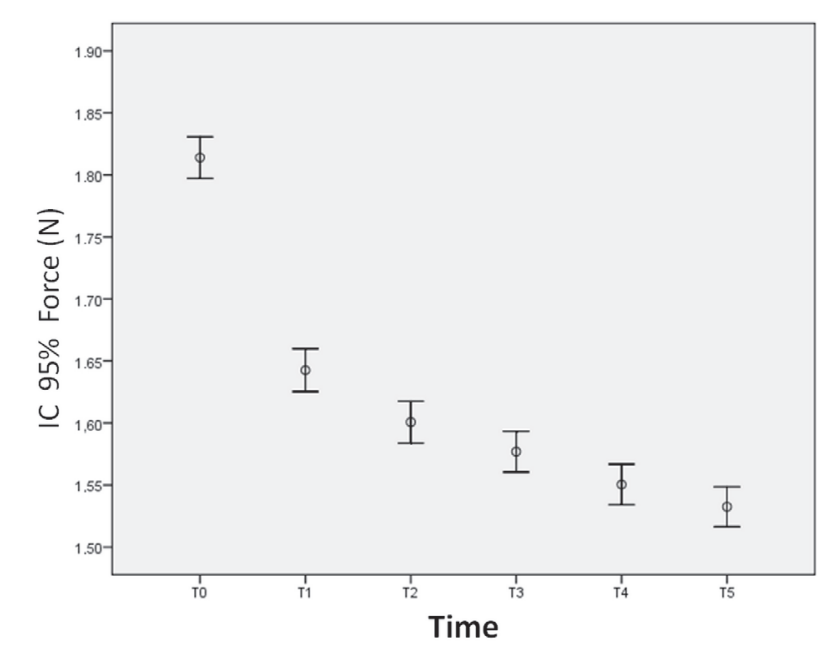

Figure 4- Mean force values of the intermaxillary elastics regardless of the group

Figure 4 exhibits the mean force values produced by the intermaxillary elastics regardless of their groups. Notice that in the first moments (from T0 to T1), there was a greater reduction of the generated forces $(p<0.05)$, followed by a decrease of degradation rate in the following periods.

This same behavior pattern was seen in the tensile strength forces produced by each group at all observation periods (Figure 5 ). Figure 5 reveals that the different groups behaved similarly at each study period ( $T 1, T 2, T 3, T 4$ and $T 5)(p>0.05)$.

\section{DISCUSSION}

This study evaluated in vitro the effects of frequently ingested beverages on the force degradation of intermaxillary elastics. The force degradation of intermaxillary elastics, measured by tensile strength test, was observed at all periods, regardless of the group (Figure 4). The greatest degree of force degradation occurred at the initial periods (from TO to T1) and a gradual reduction of this degradation occurred at the following periods, showing a tendency to reduction over time. This was confirmed by the statistically significant difference 
existing between $\mathrm{T} 0$ and $\mathrm{T} 1, \mathrm{~T} 1$ and $\mathrm{T} 2, \mathrm{~T} 2$ and $\mathrm{T} 4$, T3 and T5 $(p<0.05)$.

The mechanical behavior of intermaxillary elastics in the present study was similar to the findings of previous studies ${ }^{3-5,8,12,15}$ in which a greater degradation in the elastic forces at the initial periods of assessment was detected. However, Fernandes, et al. ${ }^{3}$ (2011) found a different behavior of the degradation of elastic forces: after a first phase of greater force degradation, there was a second stage where the elastics partially recovered the force (negative degradation), followed by a third stage in which slow and gradual force degradation occurred.

Due to this behavior, Andreassen and Bishara ${ }^{1}$ (1970) suggested the use of a force magnitude four times greater than the intended magnitude for a determined case. Consequently, when the elastics lose $40 \%$ of their initial force, levels close to those required would still be present for longer periods. Also, this would allow less frequent elastic changes. Notwithstanding, such procedure should be carefully analyzed because of the risks of damaging the tooth and periodontal tissues by excessively strong forces. Additionally, the findings of an in vivo study ${ }^{15}$ suggested that elastics should be changed daily because the oral activity dynamics would increase the fatigue and deformation of material, enhancing the risk of their fracture and failure.

Some in vitro studies have been conducted using a dynamic experimental model in which mouth opening and closing movements are simulated, stretching the intermaxillary elastics. For the present study, a static experimental model was chosen based on the results of previous investigations ${ }^{5,14}$, which demonstrated that the cyclic elastic stretching only enhances force degradation in the first hour of the experiment; after this period, the degradation rate returns to its pattern and, by the end of the study, cyclic stretching or not exerts no statistically significant influence on the elastics ${ }^{5}$. Liu, et al. ${ }^{8}$ (1993) showed that after 200 stretching cycles there was no significant force degradation of intermaxillary elastics.

The results of this research indicate that the contact of dietary beverages with intermaxillary elastics is not able to influence the degradation degree of their forces (Figure 5). The elastics presented the same behavior when immersed in either artificial saliva or test beverages at all observational periods. The lack of influence of dietary components on the degradation of intermaxillary forces has also been observed in a laboratory study ${ }^{2}$. Other studies ${ }^{6,12}$ have demonstrated that the $\mathrm{pH}$ of the medium in which the intermaxillary elastics are immersed did not influence significantly force decrease of the elastics. Based on this evidence, the beverages used in the present study were not subjected to any $\mathrm{pH}$ analysis.

The mechanical behavior of the intermaxillary elastics in the present study can be explained by the "swelling phenomenon". The chemically cross-linked points among the molecular chains enable the absorption of liquid into the latex threedimensional reticulate structure. The rubber's free energy decreases with the simultaneous effect of mixing entropy. At the same time, the molecular chains are elongated followed by the decrease of elasticity of the rubber ${ }^{15}$.

Although this study showed solid evidences, the obtained results should be carefully examined because no in vitro study is capable of simulating all factors acting on intermaxillary elastics in the oral environment. Notwithstanding, our findings contribute to improve the knowledge of the mechanical behavior of the intermaxillary elastics employed in Orthodontics, enabling greater predictability in the application of mechanotherapy involving latex elastics. The results also have clinical applications since no restrictions were found to the consumption of the studied beverages in relation to the use of the tested intermaxillary elastics, bringing comfort and freedom for patients choosing their own diet during the orthodontic treatment.

\section{CONCLUSIONS}

The chemical nature of the evaluated beverages was not able of influencing the degree of degradation of intermaxillary elastics' forces.

\section{REFERENCES}

1- Andreasen GF, Bishara S. Comparison of alastik chains with elastics involved with intra-arch molar to molar forces. Angle Orthod. 1970;40:151-8.

2- Beattie S, Monaghan P. An in vitro study simulating effects of daily diet and patient elastic band change compliance on orthodontic latex elastics. Angle Orthod. 2004;74:234-9.

3- Fernandes DJ, Fernandes GM, Artese F, Elias CN, Mendes AM. Force extension relaxation of medium force orthodontic latex elastics. Angle Orthod. 2011;81:812-9.

4- Gioka C, Zinelis S, Eliades T, Eliades G. Orthodontic latex elastics: a force relaxation study. Angle Orthod. 2006;76:475-9. 5- Kanchana P, Godfrey K. Calibration of force extension and force degradation characteristics of orthodontic latex elastics. Am J Orthod Dentofacial Orthop. 2000;118:280-7.

6- Kersey ML, Glover KE, Heo G, Raboud D, Major PW. A comparison of dynamic and static testing of latex and nonlatex orthodontic elastics. Angle Orthod. 2003;73:181-6.

7- Lacerda dos Santos R, Pithon MM, Romanos MT. The influence of $\mathrm{pH}$ levels on mechanical and biological properties of nonlatex and latex elastics. Angle Orthod. 2012;82:709-14.

8- Liu CC, Wataha JC, Craig RG. The effect of repeated stretching on the force decay and compliance of vulcanized cis-polyisoprene orthodontic elastics. Dent Mater. 1993;9:37-40.

9- Nattrass C, Ireland AJ, Sherriff M. The effect of environmental factors on elastomeric chain and nickel titanium coil springs. Eur J Orthod. 1998;20:169-76. 
10- Santos RL, Pithon MM, Martins FO, Romanos MT, Ruellas AC. Cytotoxicity of latex and non-latex orthodontic elastomeric ligatures on L929 mouse fibroblasts. Braz Dent J. 2010;21:205-10. 11- Santos RL, Pithon MM, Silva Mendes G, Romanos MT, Oliveira Ruellas AC. Cytotoxicity of intermaxillary orthodontic elastics of different colors: an in vitro study. J Appl Oral Sci. 2009;17:326-9. 12- Sauget PS, Stewart KT, Katona TR. The effect of $\mathrm{pH}$ levels on nonlatex $v s$ latex interarch elastics. Angle Orthod. 2011;81(6):1070-4
13- Stevenson JS, Kusy RP. Force application and decay characteristics of untreated and treated polyurethane elastomeric chains. Angle Orthod. 1994;64:455-64; discussion 457-65.

14- Teixeira L, Pereira BR, Bortoly TG, Brancher JA, Tanaka OM, Guariza-Filho $\mathrm{O}$. The environmental influence of Light Coke, phosphoric acid, and citric acid on elastomeric chains. J Contemp Dent Pract. 2008;9:17-24.

15- Wang T, Zhou G, Tan X, Dong Y. Evaluation of force degradation characteristics of orthodontic latex elastics in vitro and in vivo. Angle Orthod. 2007;77:688-93. 